\title{
¿Hay correlación entre hallazgos de resonancia magnética y artroscopia en lesiones meniscales de rodilla?
}

\author{
Is there a correlation between findings by MRI and \\ arthroscopy in knee meniscal lesions? \\ Rubén García Linage, ${ }^{*}$ Minseung Jeong, ${ }^{*}$ Eric Misael Saucedo Moreno ${ }^{\ddagger}$ \\ Citar como: García LR, Jeong M, Saucedo MEM. ¿Hay correlación entre hallazgos de resonancia magnética y artroscopia \\ en lesiones meniscales de rodilla? Acta Med. 2021; 19 (1): 35-39. https://dx.doi.org/10.35366/98567
}

\section{Resumen}

En la detección de las lesiones meniscales, es útil la clínica y, como complemento de análisis, se sugiere utilizar la resonancia magnética nuclear (RMN), la cual permite profundizar en la articulación y describir topográficamente el tipo de traumatismo que tiene el menisco. No obstante, existen situaciones en las cuales aparece el menisco intacto en la RMN estando roto, o viceversa. Estudio prospectivo, longitudinal, descriptivo, con datos de expedientes de pacientes que habían sido operados en el periodo de marzo de 2013 a febrero de 2018, en un hospital de tercer nivel del sector privado en la Ciudad de México. Se incluyeron sujetos mayores de 18 años de edad, de cualquier sexo, con diagnóstico de lesión de menisco, unilateral o bilateral, que fueron intervenidos de forma artroscópica. El dictamen se realizó mediante resonancia magnética y se comparó con artroscopia. La RMN, ante las lesiones de menisco medial, muestra baja especificidad $(47.6 \%)$ y sensibilidad (58.8\%) en comparación con diversas investigaciones a nivel mundial; los autores estiman que podría ser por el tipo de gabinete y la interpretación. En cambio, en las lesiones de menisco lateral, tiene baja sensibilidad (10.35\%) y muy alta especificidad (92\%). Se encontró un valor predictivo negativo (VPN) de $89.7 \%$ para lateral y $47.8 \%$ para medial.

Palabras clave: Artroscopia de rodilla, lesión meniscal, resonancia magnética de rodilla.

\section{Abstract}

In the detection of meniscal injuries, the clinic is useful and, as a supplementary study, it is suggested to use nuclear magnetic resonance imaging (MRI), which allows a deeper look of the joint and topographically describe the type of injury in the meniscus. However, there are situations in which the meniscus appears intact in the MRI and broken in the arthroscopy. Prospective, longitudinal, descriptive study, with data from records of patients that were intervened in a period from March 2013 to February 2018, at a third-level private sector hospital in Mexico City. Subjects over 18 years of age, of any gender, diagnosed with meniscus injury, unilateral or bilateral, who were operated arthroscopically where included. The diagnosis was made using magnetic resonance imaging and compared with arthroscopy. MRI in medial meniscus injuries has low specificity $(47.6 \%)$ and sensitivity (58.8\%) compared to various studies worldwide; the authors believe this could be because of the interpretation. In contrast, in lateral meniscus injuries, it has low sensitivity $(10.35 \%)$ and high specificity $(92 \%)$. The NPV found was $89.7 \%$ for lateral meniscus and $47.8 \%$ for medial.

Keywords: Knee arthroscopy, meniscal injury, knee nuclear magnetic resonance imaging.

* Médico Residente de cuarto año de Ortopedia y Traumatología. Facultad Mexicana de Medicina de la Universidad La Salle.

‡ Cirugía General y Asesor Estadístico.

Correspondencia:

Rubén García Linage

Correo electrónico: rglinagemd@gmalil.com

Aceptado: 14-04-2020.

Hospital Ángeles Mocel. Ciudad de México, México.

www.medigraphic.com/actamedica 


\section{INTRODUCCIÓN}

Los meniscos son estructuras fibrocartilaginosas que se encuentran en la superficie articular de la tibia. Tienen una disposición de medialuna, aunque el menisco medial presenta una curvatura más abierta que el lateral. Éstos se dividen en cinco zonas distintas, cuerno anterior, segmento anterior, segmento medio o cuerpo, segmento posterior y cuerno posterior. $^{1}$

En la detección de las lesiones meniscales, es útil la clínica y, como complemento de estudio, se sugiere utilizar la resonancia magnética nuclear (RMN), la cual permite profundizar en la articulación y describir topográficamente el tipo de daño que tiene el menisco. No obstante, existen situaciones en las cuales aparece el menisco intacto en la RMN si está roto, o viceversa. La importancia en la planificación preoperatoria por RMN es crucial, en caso de tener una lesión por asa de balde, se requiere tener material preparado antes del momento quirúrgico..$^{2,3}$

Para quien interpreta este examen, uno de los grandes retos es descartar estos traumatismos, es difícil ver el signo de "ausencia de la corbata de moño" y frecuentemente pasan desapercibidos. ${ }^{4}$

En el diagnóstico de lesiones meniscales, se deben dividir las laterales de las mediales, pues el comportamiento es distinto. El menisco lateral es más laxo, esto hace que presente menos daños que el menisco medial. Bin $\mathrm{S}$ encontró que el menisco medial es más sensible en RMN que el menisco lateral, específicamente, las lesiones del cuerno posterior. ${ }^{5}$

Es cierto que la RMN es el análisis de elección hoy en día para el diagnóstico de daños intraarticulares de tejido blando en rodilla, pero existen ciertos factores bien estudiados que provocan errores al momento del diagnóstico imagenológico, se debe tomar en cuenta el aparato que se esté utilizando, así como los milímetros de cortes que dé la imagen, de ser muy gruesos, pueden pasar desapercibidos, otra consideración muy importante es quién interprete estas imágenes, a más años de entrenamiento, mayor sensibilidad de diagnóstico ante lesiones meniscales. ${ }^{6}$

El objetivo del trabajo es demostrar que existe una gran diferencia entre los diagnósticos de lesiones de menisco realizados por resonancia magnética y los vistos en las artroscopia, generalmente el diagnóstico radiológico no es el mismo al diagnóstico artroscópico.

Existe un gran sesgo en la interpretación de ambos métodos, ya que quien lo descifra es el especialista al momento de hacer el examen.

\section{MATERIAL Y MÉTODOS}

Estudio prospectivo, longitudinal, descriptivo, con datos de expedientes de pacientes que fueron operados en un periodo de marzo de 2013 a febrero de 2018, en un hospital de tercer nivel del sector privado en la Ciudad de México. Se incluyeron sujetos mayores de 18 años de edad, de cualquier sexo, con diagnóstico de lesión de menisco, unilateral o bilateral, que fueron intervenidos de forma artroscópica. Se excluyeron pacientes menores de 18 años y que además presentaran traumatismo del ligamento cruzado anterior. El dictamen se realizó mediante resonancia magnética nuclear y se comparó con artroscopia, con la intención de reconocer si la resonancia magnética (RM) preoperatoria tiene adecuada sensibilidad, especificidad, valor predictivo positivo (VPP) y VPN, a fin de diagnosticar lesiones de menisco. Cabe mencionar que los datos y hallazgos transoperatorios fueron recolectados por un médico ortopedista que no tenía conocimiento del resultado de la RM previa.

El análisis estadístico se realizó con el software SPSS versión 20.0 (SPSS, Chicago, EUA), se realizó un análisis de Kolmogórov-Smirnov para determinar la distribución de las variables cuantitativas, con base en dicha distribución se resumieron mediante media y desviación estándar, fueron analizados mediante prueba $\chi^{2}$, se obtuvo la sensibilidad, especificidad VPP y VPN mediante tablas de contingencia, se consideró con un valor significativo de $\mathrm{p}$ $=0.05$ con un IC $95 \%$.

\begin{tabular}{lc}
\hline \multicolumn{2}{|c}{ Tabla 1: Variables generales. } \\
\hline Variable & $\mathrm{n}(\%)$ \\
\hline Edad, media \pm DE & $42.36 \pm 16.3$ \\
Género femenino & $323(52.3)$ \\
Lesión por RM & \\
$\quad$ Lateral & $61(9.9)$ \\
Medial & $346(56.0)$ \\
Bilateral & $211(34.1)$ \\
Tiempo de evolución & \\
Agudo & $284(45.7)$ \\
Completo & $334(54.3)$ \\
Mecanismo de lesión & $381(61.7)$ \\
Postraumático & $126(20.4)$ \\
Degenerativo & $60(9.7)$ \\
Mecánico & $36(5.8)$ \\
Postquirúrgico & $6(1.0)$ \\
Post-esfuerzo & $6(0.6)$ \\
Idiopático & $2(0.3)$ \\
Inflamatorio & $2(0.3)$ \\
Infeccioso & $1(0.16)$ \\
Congénito & \\
\hline DE = desviación estándar; RM = resonancia magnética.
\end{tabular}

DE = desviación estándar; RM = resonancia magnética. 


\section{RESULTADOS}

Se incluyeron 696 pacientes en la investigación, de los cuales se excluyeron 78 pacientes por presentar lesión de ligamento cruzado anterior (LCA) y no estar de acuerdo con el protocolo. Se estudiaron un total de 618 pacientes, de los cuales el $52.3 \%$ de esa cantidad fue del género femenino $(n=323)$. Con una media de edad de 42.36 años $( \pm 16.3)$. El 9.9\% $(n=61)$ de los pacientes presentaron lesión lateral de menisco por RM, el 56.0\% $(n=346)$ lesión medial y el $34.1 \%(n=211)$ lesión bilateral. El tiempo de evolución fue, en su mayoría, crónico con un $54.3 \%$ de los casos ( = 334) (Tabla 1).

Al comparar los resultados de la RM con los hallazgos transoperatorios, se identificó que, para las lesiones de menisco medial, la resonancia magnética tiene una sensi- bilidad de $58.8 \%($ IC $95 \%=53.6$ a $63.9 \%)$, la especificidad de $47.6 \%$ (IC 95\% = 41.8 a $53.5 \%$ ) (Tabla 2).

En lesiones laterales de menisco se identificó una pobre sensibilidad de la RM para el diagnóstico, con un $10.3 \%$ (IC 95\% = 5.1 a 19.8\%); sin embargo, los valores de especificidad son de 92\% (IC 95\% = 89.4 a 94\%) (Tabla 3).

De igual forma, tratándose de lesiones bilaterales de menisco, se encontraron valores muy pobres en sensibilidad de la RM de $37.4 \%$ (IC 95\% = 31.2 a 44.1\%), especificidad de $67.8 \%$ (IC 95\% = 63.1 a $72.2 \%)$ (Tabla 4).

\section{DISCUSIÓN}

Dentro del presente trabajo, se encontró que las lesiones de menisco medial son más comunes, las cuales son cau-

\begin{tabular}{|c|c|c|c|}
\hline & Artroscopia positiva & Artroscopia negativa & Total \\
\hline Resonancia magnética positiva & 203 & 143 & 346 \\
\hline Resonancia magnética negativa & 142 & 130 & 272 \\
\hline \multirow[t]{2}{*}{ Total } & 345 & 273 & 618 \\
\hline & $\%$ & IC 95\% & \\
\hline Sensibilidad & 58.8 & 53.6 a 63.9 & \\
\hline Especificidad & 47.6 & 41.8 a 53.5 & \\
\hline VPP & 58.7 & 53.4 a 63.7 & \\
\hline VPN & 47.8 & 41.9 a 53.9 & \\
\hline Exactitud & 53.9 & 49.9 a 57.8 & \\
\hline
\end{tabular}

Tabla 3: Tabla de contingencia en lesiones laterales.

\begin{tabular}{|c|c|c|c|}
\hline & Artroscopia positiva & Artroscopia negativa & Total \\
\hline Resonancia magnética positiva & 7 & 44 & 51 \\
\hline Resonancia magnética negativa & 61 & 506 & 567 \\
\hline \multirow[t]{2}{*}{ Total } & 68 & 550 & 618 \\
\hline & $\%$ & IC 95\% & \\
\hline Sensibilidad & 10.3 & 5.1 a 19.8 & \\
\hline Especificidad & 92.0 & 89.4 a 94.0 & \\
\hline VPP & 13.7 & 6.8 a 25.7 & \\
\hline VPN & 89.7 & 80.2 a 94.9 & \\
\hline Exactitud & 83.0 & 79.8 a 85.8 & \\
\hline
\end{tabular}


Tabla 4: Tabla de contingencia para lesiones bilaterales.

\begin{tabular}{|c|c|c|c|}
\hline & Artroscopia positiva & Artroscopia negativa & Total \\
\hline Resonancia magnética positiva & 79 & 131 & 210 \\
\hline Resonancia magnética negativa & 132 & 276 & 408 \\
\hline \multirow[t]{2}{*}{ Total } & 211 & 407 & 618 \\
\hline & $\%$ & IC $95 \%$ & \\
\hline Sensibilidad & 37.4 & 31.2 a 44.1 & \\
\hline Especificidad & 67.8 & 63.1 a 72.2 & \\
\hline VPP & 37.6 & 31.3 a 44.3 & \\
\hline VPN & 67.6 & 63.0 a 72 & \\
\hline Exactitud & 57.4 & 53.5 a 61.3 & \\
\hline
\end{tabular}

sadas por un mecanismo crónico y postraumático, lo cual concuerda con los resultados que se obtuvieron en cuanto a la especificidad y sensibilidad de lesiones mediales.

Al parecer, las lesiones de menisco lateral son un reto mayor en el diagnóstico de imagen, ya que se encontraron muchas por artroscopia, más que las reportadas en la RMN.

Christopher S. Lee encontró, en un estudio de cohorte de 379 pacientes, donde la mayoría fueron hombres, con edad promedio de 47.5 años, una especificidad, sensibilidad, VPP y VPN de 83, 81, 89 y $73 \%$ respectivamente para menisco medial. En el menisco lateral las cifras fueron de 51, 93, 84 y $73 \% .^{7}$ Al compararse con el presente estudio, se tuvieron resultados más elevados en cuanto a porcentaje, pero coinciden con la dificultad para realizar el dictamen por imagen del menisco lateral.

Nasil Singh describe, en su reporte, un total de 39 pacientes, donde 26 fueron femeninos y 13 masculinos, con edades entre 18 a 69 años, un valor predictivo negativo de $81 \%$ en el menisco medial y $90 \%$ para menisco lateral. No refiere un mecanismo especifico de lesión. ${ }^{8}$ En este trabajo se encontró que, del total de los pacientes, el 52.3\% fueron femeninos, con una edad media de 43.36 años, donde el mecanismo más común fue postraumático, un VPN 89.7 para lateral y 47.8 en el medial.

En el 2019, Bruno F. publicó que la RMN tiene una sensibilidad del $98.5 \%$ y especificidad del $94.7 \%$ para el menisco interno, en cuanto al menisco externo, tiene sensibilidad de $98.5 \%$ y especificidad de $90.5 \%$. En esta investigación, para menisco medial fue de 58.8 y $47.6 \%$ respectivamente y en el menisco lateral de 10.3 y $92 \% .^{9}$

Tanto en los estudios previos como en el presente, se encontró que el traumatismo más frecuente es el del menisco medial. En cuanto a los resultados de este trabajo, se hallaron una sensibilidad y especificidad más bajas. Los autores opinan que se debe a que en esta experiencia el gabinete no se hizo en el mismo centro hospitalario y fueron interpretados por diferentes radiólogos con distintos tipos de resonadores, la muestra podría ser más grande y unificar los resultados para que sean interpretados por el mismo radiólogo, de la misma forma las artroscopias deben ser realizadas por el mismo cirujano.

La RMN sigue siendo un método diagnóstico útil, ya que no hay ningún estudio a nivel global que demuestre mejores resultados, se encontró que influye mucho la experiencia de quien la interprete. La importancia de estos hallazgos es entender que, en esta población, se debe hacer uso de la clínica como herramienta principal, y no basarse exclusivamente en la MRI, por las diferencias que se pueden encontrar. Se debe hacer un estudio prospectivo para encontrar más resultados.

\section{CONCLUSIONES}

Después de analizar los resultados, se observó que la mayoría de los pacientes con traumatismos de menisco fueron mujeres adultas con mecanismo postraumático, quienes se presentan con lesiones crónicas.

La lesión más común es la del menisco medial, seguida por la bilateral y finalmente la lateral aislada.

La RMN, ante las lesiones de menisco medial, evidencia baja especificidad y sensibilidad en comparación con diversos reportes a nivel mundial, probablemente es por el tipo de gabinete y la interpretación. En cambio, en los daños de menisco lateral, presenta baja sensibilidad y muy alta especificidad.

La clínica es la herramienta principal ante las lesiones meniscales. 


\section{REFERENCIAS}

1. Pujol N, Boisrenoult $P$, Beaufils P. Lesiones traumáticas de los meniscos de la rodilla. EMC - Aparato Locomotor. 2014; 47 (2): 1-10.

2. De Smet AA, Graf BK. Meniscal tears missed on MR imaging: relationship to meniscal tear patterns and anterior cruciate ligament tears. AJR Am J Roentgenol. 1994; 162 (4): 905-911.

3. Wright DH, De Smet AA, Norris M. Bucket-handle tears of the medial and lateral menisci of the knee: value of MR imaging in detecting displaced fragments. AJR Am J Roentgenol. 1995; 165 (3): 621-625.

4. Helms CA, Laorr A, Cannon WD Jr. The absent bow tie sign in buckethandle tears of the menisci in the knee. AJR Am J Roentgenol. 1998; 170 (1): 57-61.
5. Bin SI, Kim JM, Shin SJ. Radial tears of the posterior horn of the medial meniscus. Arthroscopy. 2004; 20 (4): 373-378.

6. Bolog NV, Andreisek G. Reporting knee meniscal tears: technical aspects, typical pitfalls and how to avoid them. Insights Imaging. 2016; 7 (3): 385-398.

7. Lee CS, Davis SM, McGroder C, Stetson WB, Powell SE. Analysis of low-field magnetic resonance imaging scanners for evaluation of knee pathology based on arthroscopy. Orthop J Sports Med. 2013; 1 (7): 2325967113513423.

8. Singh N, Hanekom H, Suleman FE. The accuracy of magnetic resonance imaging diagnosis of non-osseous knee injury at Steve Biko Academic Hospital. SA J Radiol. 2019; 23 (1): 1754.

9. Bruno F, Goderecci R, Barile A, Calvisi V. Comparative evaluation of meniscal pathology: MRI vs arthroscopy. J Biol Regul Homeost Agents. 2019; 33 (2 Suppl. 1): 9-14. 